\title{
BMJ Open New geographic model of care to manage the post-COVID-19 elective surgery aftershock in England: a retrospective observational study
}

\author{
Jonathan Clarke (D) , ${ }^{1}$ Alice Murray, ${ }^{2}$ Sheraz Rehan Markar (D) ,' \\ Mauricio Barahona (D) , ${ }^{1}$ James Kinross, ${ }^{2}$ on behalf of The PanSurg Collaborative
}

To cite: Clarke J, Murray A, Markar SR, et al. New geographic model of care to manage the post-COVID-19 elective surgery aftershock in England: a retrospective observational study. BMJ Open 2020;10:e042392. doi:10.1136/ bmjopen-2020-042392

- Prepublication history and additional materials for this paper is available online. To view these files, please visit the journal online (http://dx.doi. org/10.1136/bmjopen-2020042392).

$\mathrm{JC}$ and $\mathrm{AM}$ contributed equally.

Received 06 July 2020

Revised 29 September 2020

Accepted 30 September 2020

A) Check for updates

(c) Author(s) (or their employer(s)) 2020. Re-use permitted under CC BY. Published by BMJ.

${ }^{1}$ Department of Mathematics, Imperial College of Science, Technology and Medicine,

London, UK

${ }^{2}$ Department of Surgery and Cancer, Imperial College of Science, Technology and Medicine, London, UK

Correspondence to Dr Jonathan Clarke; j.clarke@imperial.ac.uk

\section{ABSTRACT}

Objectives The suspension of elective surgery during the COVID-19 pandemic is unprecedented and has resulted in record volumes of patients waiting for operations. Novel approaches that maximise capacity and efficiency of surgical care are urgently required. This study applies Markov multiscale community detection (MMCD), an unsupervised graph-based clustering framework, to identify new surgical care models based on pooled waiting-lists delivered across an expanded network of surgical providers.

Design Retrospective observational study using Hospital Episode Statistics.

Setting Public and private hospitals providing surgical care to National Health Service (NHS) patients in England.

Participants All adult patients resident in England undergoing NHS-funded planned surgical procedures between 1 April 2017 and 31 March 2018.

Main outcome measures The identification of the most common planned surgical procedures in England (highvolume procedures (HVP)) and proportion of low, medium and high-risk patients undergoing each HVP. The mapping of hospitals providing surgical care onto optimised groupings based on patient usage data.

Results A total of 7811891 planned operations were identified in 4284925 adults during the 1-year period of our study. The 28 most common surgical procedures accounted for a combined 3907474 operations (50.0\% of the total). $2412613(61.7 \%)$ of these most common procedures involved 'low risk' patients. Patients travelled an average of $11.3 \mathrm{~km}$ for these procedures. Based on the data, MMCD partitioned England into 45, 16 and 7 mutually exclusive and collectively exhaustive natural surgical communities of increasing coarseness. The coarser partitions into 16 and seven surgical communities were shown to be associated with balanced supply and demand for surgical care within communities.

Conclusions Pooled waiting-lists for low-risk elective procedures and patients across integrated, expanded natural surgical community networks have the potential to increase efficiency by innovatively flexing existing supply to better match demand.
Strengths and limitations of this study

- The COVID-19 pandemic has significantly disrupted the provision of planned surgical care in hospitals across the world. Addressing the accumulated backlog of cases requires a new model of care whereby procedures are carried out at pace, while also responding to the dynamic risk of further COVID-19 outbreaks.

- This study uses national, retrospective hospital administrative data relating to 7.8 million interventional procedures in 4.2 million adults.

- Markov multiscale community detection, an unsupervised network clustering technique, is applied to understand how providers of surgical care may collaborate with one another based on prior patterns of surgical care delivery.

- The relative imbalances in supply and demand for surgical care within the identified surgical communities are quantified in order to determine the potential applicability of different scales of collaboration between care providers.

- While this study advances the potential role of collaboration between surgical centres to address the surgical backlog resulting from COVID-19, it does not address issues relating to local financial or logistical barriers to implementation of such a strategy.

\section{INTRODUCTION}

The COVID-19 pandemic put a global halt to the majority of elective surgery in order to manage the surge in patients requiring acute hospital services and intensive treatment unit care ${ }^{1-4}$ It has been estimated that 28 million elective operations worldwide have been cancelled or postponed due to the pandemic. ${ }^{5}$ Although the focus of public health organisations globally was rightly mounting an effective emergency response to the COVID-19 pandemic, the surgical 'aftershock' will therefore be unprecedented and yet to be fully appreciated. Millions of patients in the UK are already waiting for treatment 
and numbers increase daily as the diversion of resources continues. ${ }^{6}$ Elective surgical services are gradually being reintroduced, aiming to treat waiting patients without risking the spread of COVID-19. Management strategies in the UK are currently focused on undertaking lifesaving cancer operations in 'clean' COVID-free hospitals or in hospital sites away from the acute care sites where COVID-19 is more prevalent. ${ }^{78}$ An immediate response to 'catch up' and clear case load will need to be undertaken, as well as adjusting to a 'new normal'.

Waiting-list numbers vary widely across the country and waiting times have increased in recent years. ${ }^{9}$ To add complexity, there is also regional variation in the number of COVID-19 infections and burden of COVIDrelated workload. ${ }^{10} 11$ Therefore, in order to respond to the needs of a particular population, dynamic, flexible and regional solutions will be required to balance the reintroduction of services with careful COVID-19 management.

Flexibility in the location where care is provided, according to patients' clinical needs, has the potential to better match supply of services where there is appropriate demand. Patients can be treated more promptly if surgeons, hospitals and hospital delivery systems work together across provider networks, managing a centrally pooled workload. While some patients will need to be treated at specific locations (particularly high-risk patients or those requiring complex cancer care), there are other less complex procedures that could feasibly be performed by a range of qualified providers for patients who are able to travel. ${ }^{12}$

As the National Health Service (NHS) in England moves towards greater integration, there is an opportunity to break down arbitrary geographic boundaries and funding barriers, and bring together multiple providers of surgical care into 'surgical communities'. In such configurations, hospitals share a centrally managed waitinglist for routine surgical procedures, and patients may receive surgery at any centre within the community of providers with the capacity to do so. There is a precedent for this approach, as a similar scheme was successfully piloted on a small scale in London. ${ }^{13}$ Pooling available capacity between communities of surgical care providers may enable the efficient use of their collective available resources.

In this study we explore the potential of using flexible locations of care as a strategy to manage waiting-lists. First, we categorise the types of elective procedures and eligible patients into groups that would be amenable to undergoing surgery in any suitable location. Second, we identify from patient data existing community networks of surgical providers ('surgical communities') that collectively provide planned surgical care to similar geographic patient populations. Third, we map these surgical communities against existing organisational configurations and model the effect on supply and demand when patients travel further for care.

\section{METHODS}

All planned inpatient admissions to hospitals in England involving a surgical procedure were identified for adults resident in England from Hospital Episode Statistics from 1 April 2017 to 31 March 2018. NHS-funded procedures conducted in non-NHS hospitals were included. For each admission, the first operative day was defined as the first day within an admission in which a surgical procedure was recorded. Procedures performed after the first operative day were excluded from the analysis. Where multiple procedures were performed on the first operative day, all of those procedures were counted to capture the fullest reliable representation of planned surgical activity. Inclusion of procedures after the first operative day is likely to include unplanned operations arising from surgical complications which are not identifiable as unplanned procedures in the data available.

All procedure codes describing diagnostic imaging, testing or rehabilitation (OPCS Classification of Intervention and Procedures version-4 codes beginning with $\mathrm{U})$, the method of a procedure $(\mathrm{Y})$ and site of a procedure $(\mathrm{Z})$ were removed in addition to miscellaneous operations (X). ${ }^{14}$ Procedures involving the concurrent extraction of a lens (C71) and insertion of a prosthetic lens (C75) were treated as a single procedure. Lower gastrointestinal (GI) diagnostic and therapeutic endoscopies frequently occurred concurrently or under codes with similar descriptions and were therefore grouped together. Conversely, diagnostic upper GI endoscopy (G45) was far more common than therapeutic endoscopies and was therefore treated separately.

\section{Classification of operative risk}

For each procedure, the age of the patient at the time of surgery was extracted. The modified Charlson comorbidity score of each patient was determined based on the presence of International Classification of Diseases 10th Revision diagnosis codes extracted from their operative admission and all other recorded admissions to hospital for each patient in the 6 months prior to surgery. ${ }^{15}$ Patients were then classified according to low, medium or high risk (for potential morbidity and mortality) by virtue of their age and Charlson score (table 1).

\section{Identification of high-volume procedures}

The total number of procedures performed for each three-digit OPCS-4 code was calculated and sorted in

Table 1 Classification of low, medium and high-risk patients based on age and Charlson score

\begin{tabular}{lllll}
\hline \multicolumn{5}{c}{ Charlson score } \\
\cline { 3 - 5 } & & $\mathbf{0}$ & $\mathbf{1 - 2}$ & $\mathbf{3 +}$ \\
\hline \multirow{2}{*}{ Age } & $<60$ & Low & Low & Medium \\
& $60-74$ & Low & Medium & High \\
& $75+$ & Medium & High & High \\
\hline
\end{tabular}


descending order by volume. Those top procedures collectively accounting for more than $50 \%$ of the overall number of procedures were selected, and hereafter referred to as 'High Volume Procedures' (HVP).

\section{Identification of hospital sites}

The site in which a procedure was performed was identified from the SITETRET code of its associated admission. The postcodes of all sites in which procedures were performed were extracted from the site-level Estates Returns Information Collection. ${ }^{16}$ Postcodes were converted to latitude and longitude coordinates. For all sites, the straight-line distance between all sites was calculated using the haversine formula. ${ }^{17}$ Where sites were within $1 \mathrm{~km}$ of one another, they were treated as a single merged site under the code and coordinates of the highest volume provider.

\section{Calculation of distance travelled for surgery}

For each patient, the approximate location of their home was determined using the coordinates of the populationweighted centroid of their lower layer super output area (LSOA) of residence. ${ }^{18}$ LSOAs are mutually exclusive, collectively exhaustive geographic census divisions defined by the UK Office for National Statistics, of which there are 32844 in England, with a mean population of 1704 people, and is therefore similar in scale to census block groups in the USA. The straight-line distance between the population-weighted centroid of the LSOA of residence of the patient and the site in which the procedure was performed was calculated according to the haversine formula.

For each HVP, the total number of procedures performed was calculated. The number of patients classified as low, medium and high risk was calculated, along with the total number of sites undertaking the procedure and the average distance travelled for surgery. For each HVP, the total number of procedures performed by each site was calculated. To exclude providers who rarely perform a procedure, the highest volume providers who collectively accounted for $99 \%$ of procedures were identified and classified as providers of the HVP.

\section{Identification of surgical communities}

The proportion of patients presenting from each LSOA in England to each regular provider site for an HVP was calculated and a normalised cosine similarity matrix of LSOAs was computed (Equation 1).

$$
\text { similarity }_{\mathrm{AB}}=\frac{\sum_{i=1}^{n} A_{i} B_{i}}{\sqrt{\sum_{i=1}^{n} A_{i}^{2}} \sqrt{\sum_{i=1}^{n} B_{i}^{2}}}
$$

\section{Equation 1}

Calculation of cosine similarity between LSOAs. $A_{i}$ is the proportion of patients presenting to hospital site $i$ resident in LSOA A; $B_{i}$ is the proportion of patients presenting to hospital site $i$ resident in LSOA B; and $n$ is the total number of hospital sites in the data set.
This matrix quantifies the similarity of patterns of presentation for HVPs between all LSOAs in England. It can be understood as the adjacency matrix of a dense, weighted network connecting LSOAs to one another according to the similarity in their patterns of presentation to hospital for HVPs. ${ }^{19}$ This network was sparsened using the relaxed minimum spanning tree technique, a method used elsewhere in applied network science to sparsen a dense, inhomogeneous network to preserve both local and global connectivity within a network. ${ }^{20}{ }^{21}$ This sparsened network was subsequently partitioned using Markov multiscale community detection (MMCD) to produce partitions of the LSOAs according to shared patterns of presentation to hospital sites for HVPs. ${ }^{22} 23$

\section{Description of surgical communities}

The total number of procedures performed in each surgical community and the total number of hospital sites were calculated. For each sustainability and transformation partnership (STP-NHS organisational divisions of England into 44 regions responsible for developing local integration between primary and secondary care providers), the effective number of surgical communities active within its boundary was calculated using the equivalent market size (the reciprocal of the Herfindahl-Hirschman Index of market concentration) (Equation 2). ${ }^{24}$

$$
\mathrm{EMS}_{\mathrm{i}}=1 / \sum_{\mathrm{j}=1}^{\mathrm{N}} \mathrm{s}_{\mathrm{ij}}^{2}
$$

\section{Equation 2}

The equivalent market size of $\mathrm{STP}_{i}$. Here, $s_{i j}$ is the proportion of LSOAs in $\mathrm{STP}_{i}$ contained within surgical community $j$, and $N$ is the number of surgical communities in the partition.

\section{Calculation of the balance between supply and demand within} surgical communities

Surgical communities were modelled as self-contained subdivisions of England containing LSOAs contributing cases requiring surgery (demand) and hospitals providing finite surgical capacity for those services (supply). ${ }^{25}$ In this configuration, surgical procedures for patients resident within a surgical community would be performed at a hospital site spatially located within the same surgical community. Within each surgical community, surgical demand was calculated as the total number of HVP cases performed for patients resident in LSOAs within the surgical community. Supply was calculated as the total number of HVP cases performed by sites located within the geographic boundary of the surgical community. The supply-demand mismatch was calculated as the percentage difference between supply and demand for each community. The median of the absolute value of the supply-demand mismatch was determined.

\section{Patient and public involvement}

We did not directly include patient and public involvement in this study, but the database used in the study was 
Table 2 The 28 procedures accounting for more than half of all elective surgical activities in England

\begin{tabular}{|c|c|c|c|c|c|}
\hline \multirow[b]{2}{*}{ Procedure } & \multirow{2}{*}{$\begin{array}{l}\text { Total number } \\
\text { of cases }\end{array}$} & \multicolumn{3}{|l|}{ Patient risk } & \multirow{2}{*}{$\begin{array}{l}\text { Mean distance } \\
\text { travelled (km) }\end{array}$} \\
\hline & & Low risk (\%) & Medium risk (\%) & High risk (\%) & \\
\hline Lower Gl endoscopy & 937616 & 74.8 & 17.9 & 7.3 & 9.8 \\
\hline Upper GI endoscopy & 650133 & 66.9 & 22.1 & 10.9 & 9.4 \\
\hline Lens extraction+ replacement & 395445 & 33.5 & 46.5 & 20.0 & 10.9 \\
\hline Excision of skin lesion & 215608 & 55.0 & 29.5 & 15.5 & 12.7 \\
\hline Injection/aspiration joint & 142562 & 71.6 & 20.9 & 7.5 & 12.6 \\
\hline Vitrectomy & 132938 & 39.9 & 44.1 & 16.1 & 13.2 \\
\hline Cystoscopy & 130114 & 56.4 & 26.2 & 17.4 & 11.8 \\
\hline Insertion of central venous catheter & 109864 & 24.3 & 38.3 & 37.4 & 14.0 \\
\hline Coronary angiography & 105620 & 56.2 & 30.0 & 13.8 & 13.9 \\
\hline Dental extraction & 101435 & 91.6 & 5.8 & 2.5 & 11.5 \\
\hline Knee replacement & 78773 & 53.3 & 34.4 & 12.3 & 13.4 \\
\hline Bladder catheterisation or irrigation & 71552 & 42.5 & 32.7 & 24.8 & 12.8 \\
\hline Injection to bladder & 67167 & 34.3 & 29.8 & 35.9 & 11.5 \\
\hline Spinal facet joint injection & 64154 & 70.4 & 21.9 & 7.7 & 14.0 \\
\hline Cholecystectomy & 61790 & 80.5 & 13.8 & 5.7 & 11.8 \\
\hline Lymph node biopsy & 60674 & 34.8 & 34.4 & 30.8 & 14.9 \\
\hline Epidural or spinal injection & 60656 & 69.2 & 22.6 & 8.1 & 12.9 \\
\hline Inguinal hernia repair & 58943 & 72.6 & 19.9 & 7.5 & 10.9 \\
\hline Spinal nerve root injection & 58212 & 77.0 & 17.5 & 5.5 & 16.2 \\
\hline Knee meniscectomy/ meniscal repair & 57871 & 93.2 & 5.9 & 0.8 & 12.4 \\
\hline Hysteroscopy & 52360 & 90.9 & 6.4 & 2.7 & 9.9 \\
\hline Carpal tunnel release & 48245 & 70.7 & 22.2 & 7.1 & 11.0 \\
\hline $\begin{array}{l}\text { Application/ removal of internal fixation } \\
\text { of bone }\end{array}$ & 46771 & 84.6 & 11.9 & 3.5 & 15.7 \\
\hline Dental clearance & 43463 & 82.3 & 11.7 & 5.9 & 11.1 \\
\hline Partial breast excision & 41827 & 50.1 & 31.4 & 18.5 & 11.5 \\
\hline Bone marrow biopsy & 38369 & 39.8 & 34.8 & 25.5 & 15.6 \\
\hline Primary joint resurfacing & 37854 & 59.3 & 30.1 & 10.5 & 14.0 \\
\hline Cystoscopy+ resection of bladder lesion & 37458 & 17.7 & 29.8 & 52.5 & 11.2 \\
\hline
\end{tabular}

The proportion of patients classified as low, medium and high risk according to table 1 is shown, along with the mean distance travelled from a patient's LSOA of residence to the hospital site in which the procedure is performed. GI, gastrointestinal; LSOA, lower layer super output area.

released following review by a panel including patient representatives.

\section{RESULTS}

A total of 7811891 planned interventional procedures corresponding to 5718031 admissions involving 4284 925 adult patients resident in England from 1 April 2017 to 31 March 2018 were identified. These procedures were performed at 530 NHS hospital sites and 162 different private provider sites. A total of 1210 different three-digit OPCS codes were used.

Twenty-eight types of procedure in table 2 accounted for 3907474 operations, over half of all planned surgical procedures during the study period. These are denoted as HVPs. Of these HVPs, 3553649 (90.9\%) were performed in an NHS site, while 353825 (9.9\%) were performed in a non-NHS site. Collectively, diagnostic or therapeutic upper and lower GI endoscopy accounted for 1.6 million procedures $(20.3 \%)$. On average, procedures were performed on patients aged 61.4 years ( $\mathrm{SD}=16.7$ years). A total of 2636559 procedures were performed on patients with a Charlson comorbidity score of $0(67.5 \%)$, while 997765 procedures were performed on patients with a Charlson score of 1 or 2 (25.5\%) and 273150 procedures were performed on patients with a Charlson score of 3 or more $(7.0 \%)$. 

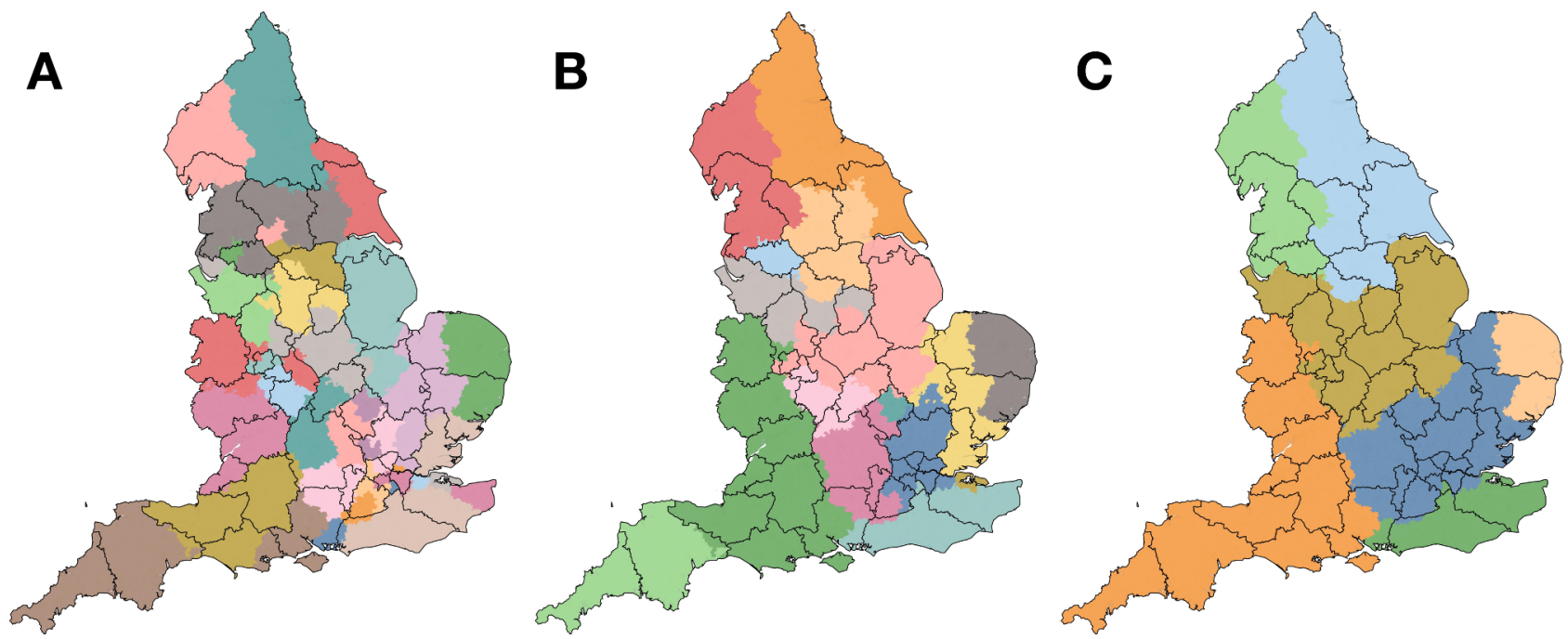

Figure 1 Division of England into 45 (panel A), 16 (panel B) and 7 (panel C) surgical communities (in colour), according to Markov stability. Sustainability and transformation partnership (STP) boundaries are overlaid (black lines).

The mean distance travelled from a patient's residence to hospital for surgery was on average $11.3 \mathrm{~km}$. Mean distances for the 28 HVPs ranged from $9.4 \mathrm{~km}$ for upper GI endoscopy to $16.2 \mathrm{~km}$ for spinal nerve root injection. A total of 2412613 (61.7\%) HVPs were performed in 'low risk' patients, 988067 (25.3\%) in 'medium risk' patients and $506794(13.0 \%)$ in 'high risk' patients. The proportion of procedures being performed on 'high risk' patients ranged from $1 \%$ for meniscal procedures to $52 \%$ for cystoscopy and resection of bladder lesions. In 22 out of $28 \mathrm{HVPs}$, more than $80 \%$ of patients were classified as 'low' or 'medium' risk.

MMCD identified (see online supplemental figure 1) three robust community conformations of LSOAs consisting of 45 (partition A), 16 (partition B) and 7 (partition C) surgical communities (figure 1). Stable spatial motifs are observed across the three partitions.

Overlaid STP boundaries show variable agreement with surgical communities (figure 1). Lower agreement is observed, for example, in East Anglia, where surgical communities consistently partition in "northsouth' direction, while the STP boundary runs 'east to west'. Close agreement can be seen in Cornwall, where STPs are adjoining, based around surgical communities. The Hampshire and Isle of Wight STP, in the south of England, remains divided between more than three surgical communities in partition $\mathrm{C}$.
The median number of HVP cases performed in each community ranges from 78998 in the finest partition (A) to 574403 in the coarsest partition (C) (table 3). In partition $\mathrm{A}$, the median number of surgical sites per community is 9 , with an IQR from 9 to 17 . In partition B, the median number of surgical sites per community is 25 , with an IQR of 19-44, while in partition C, a median of 84 surgical sites are present per community, with an IQR of 56-98. In partition A, STPs involved a median of 1.7 surgical communities, compared with 1.1 for partition B and 1.0 for partition C. Only the Hampshire and the Isle of Wight STP remains divided between more than three surgical communities in partition $\mathrm{C}$ (figure 2).

Supply and demand relationships within surgical communities In partition $\mathrm{A}$, median absolute percentage difference between supply and demand for HVPs within surgical communities is $5.1 \%$. Twelve communities $(27 \%)$ had absolute mismatches between supply and demand of more than $10 \%$ (table 3 ). These communities were located around conurbations in the North West of England and Greater London, with supply exceeding demand within cities, and demand exceeding supply in suburban communities (figure 3). In partition B, a supply-demand mismatch exceeding $10 \%$ is only observed for the surgical community on the south of the Thames Estuary, where demand exceeds supply by $25 \%$, indicating a role for

Table 3 Descriptive statistics for the three optimal partitions produced

\begin{tabular}{llll}
\hline Partition & A & B & C \\
\hline Communities $(\mathrm{n})$ & 45 & 16 & 7 \\
Median number of cases per community & $78998(43628-118087)$ & $214216(122823-314022)$ & $574403(406465-679703)$ \\
$\begin{array}{l}\text { Median number of treatment sites per } \\
\text { community }\end{array}$ & $9(5-17)$ & $25(19-44)$ & $84(56-98)$ \\
$\begin{array}{l}\text { Absolute supply:demand mismatch (\%) } \\
\text { (5.1 (2.9-10.2) }\end{array}$ & $4.1(1.0-5.7)$ & $2.2(1.0-2.9)$ \\
\hline
\end{tabular}

IQRs are shown in parentheses where appropriate. 
A

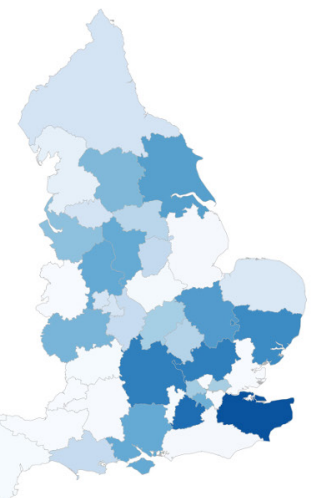

B

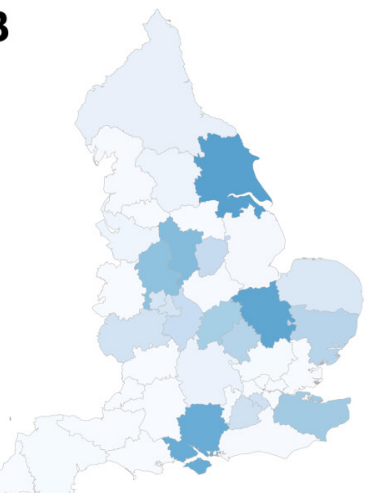

C

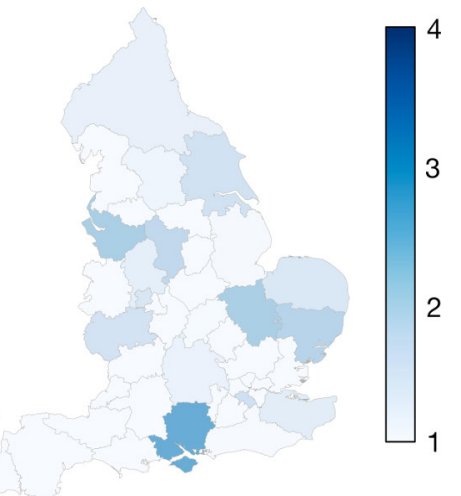

Figure 2 The equivalent number of surgical communities active in each sustainability and transformation partnership (STP) as determined by the Equivalent Market Size (EMS) for configurations of 45 (panel A), 16 (panel B) and 7 (panel C) surgical communities. Areas of darker blue, 4 (eg, East Anglia), represent those areas with greatest difference between surgical communities and STPs, whereas lighter blue shows greater agreement (eg, Cornwall).

nearby surgical sites in East London which lie outside of the community. In partition $\mathrm{C}$, the percentage difference between supply and demand does not exceed $5 \%$ in any community.

\section{DISCUSSION}

Hospital providers, policymakers and clinicians urgently require solutions for managing the COVID-19 elective surgical aftershock. This describes a state where COVID-19 cases are in decline, in the context of strategically halted elective surgery and exponentially growing waiting-lists. The extraordinary levels of demand for operations now require radical new solutions to the way we organise and deliver surgical services. This study showed that there are existing hospital networks performing high volumes of low-risk procedures for low-risk, local patients. When we compare supply and demand for planned surgical care across England, the degree of mismatch varies widely, particularly around conurbations. Importantly, these data demonstrate that variation is reduced significantly when provider networks expand and smaller surgical communities coalesce into 16 larger geographic regions. We have identified a large group of potentially eligible, fit, lower risk patients who could be asked to travel greater distances than the existing median of $13 \mathrm{~km}$ for their more minor surgery in order to shorten waiting times.

Central management of pooled waiting-lists across an increased number of both NHS and non-NHS providers offers an opportunity for greater collaboration between surgical centres and a better distribution of workload. It would provide enhanced system resilience in the context of future COVID-19 outbreaks to continue planned surgery in dedicated clean sites. ${ }^{82627}$ The scheme may have additional benefits including increased patient choice, greater workforce flexibility and maximisation of teams across areas, with increasing efficiency. There is a paucity of high-quality data on the effects of pooled waiting-lists. ${ }^{28}$ Some evidence for their potential success has come from smaller, single-site initiatives piloting internal pooling of cases distributed to consultants in the same department. ${ }^{29} 3030$ Surgical pooling has been used successfully in crises to achieve waiting-list targets with work done by non-consultant grade surgeons and cases shifted to the private sector. Surgical pooling has also
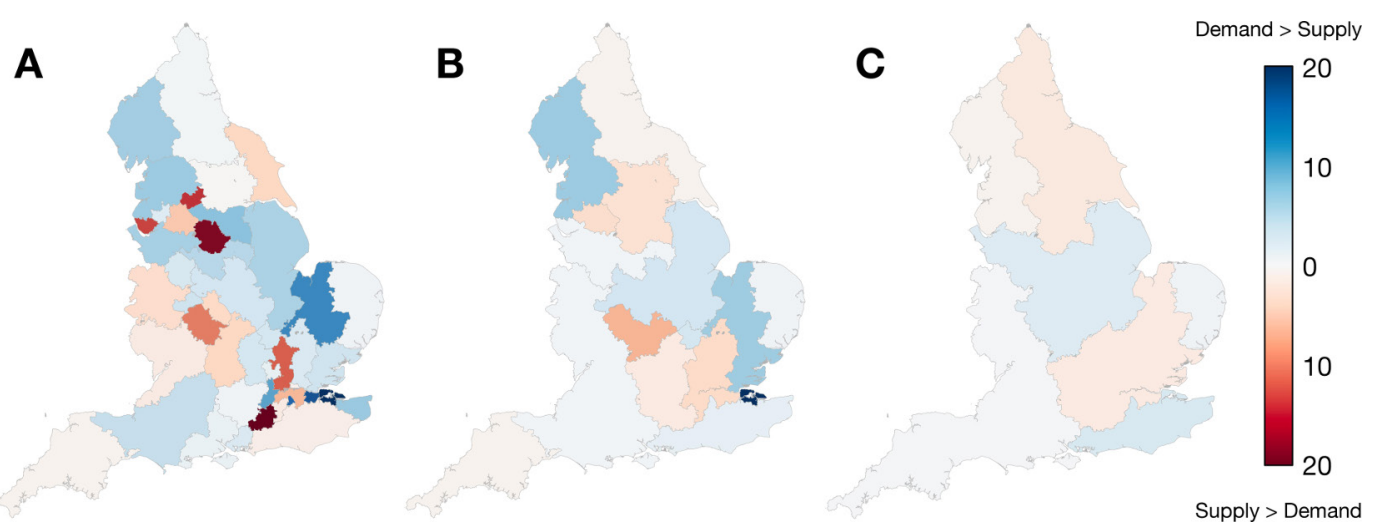

Figure 3 The absolute percentage difference between the number of patients undergoing surgery resident within a surgical community (demand) and the number of procedures performed by hospitals within the community (supply) for configurations of 45 (panel A), 16 (panel B) and 7 (panel C) surgical communities. Areas in blue represent those surgical communities where procedures performed on patients outnumber those performed by the local providers. 
been successful in matching existing supply to demand across transplant networks where donors are matched to recipients across larger regions, and sometimes between countries. ${ }^{31}$ Greater choice and increased competition between providers for patients can be associated with reduced waiting times..$^{32}$ In this study, we remain agnostic as to the means by which providers within a pooled list community should collaborate, and accept that the timing and mechanism of any collaboration should reflect the idiosyncrasies of local contexts over time-and is a determination which is best made by local providers.

The London Patient Choice Project was set up to reduce long waiting times for patients awaiting ophthalmic and minor general surgery procedures. Waiting-lists were centrally pooled, managed and funded, with patients then given a choice on-site of care in order to obtain earlier treatment. This leads to a convergence of waiting times across providers by relieving those hospitals with longer lists. ${ }^{133}$ Central purchasing of services was likely key to its success. On the strength of this pilot project, the English NHS undertook a national roll-out of patient choice, but without the central purchasing or coordination. 'Choose \& Book' offered patients a choice of at least four hospitals which led some patients to attend a hospital other than the nearest one. Unpicking the effect of Choose \& Book on waiting-lists separately to other initiatives piloted at the time is complex, but it is likely that the setting of targets and strong performance management were key drivers on reducing waiting times rather than patient choice alone. ${ }^{34}$

In the UK, patients generally favour the convenience and familiarity of a local provider. However, a MORI poll for the British Medical Association showed that if faced with a long wait, $27 \%$ of people would travel anywhere in the UK for treatment by the NHS. ${ }^{35}$ Seventy-eight per cent of patients surveyed in the Isle of Wight were willing to travel to the mainland for elective surgery where the wait was shorter. ${ }^{36}$ Greater patient travel has the potential to alleviate focal strain on services, but its practical application will require careful consideration. There are a number of barriers to travelincluding patient mobility, age and risk as well as the cost of travel and the need for nearby family and friend support. In this study, selection of 'low-risk patients and procedures' acts to mitigate some of these concerns, although the identification of operative risk based on procedure, age and Charlson score may be limited, and clearly in practice a patient-specific, case-by-case approach would be required. Government subsidisation of travel would be an important intervention to reduce inequalities based on socioeconomic status, education level, vulnerability or social exclusion. ${ }^{35}$ However, in the London pilot, there was no evidence of inequalities in uptake of the pooled list scheme by social class, educational attainment, income or ethnicity. ${ }^{13}$ In the UK, with increasing centralisation of complex care, particularly cancer care, patients are often already asked to travel further. ${ }^{37}$ The applicability of a pooled list surgical strategy varies according to the complexity of procedures and the need for in-person longitudinal follow-up with the operating centre. The finding that the majority of HVPs in this study are low-complexity procedures, with limited need for onward follow-up, supports the suitability of pooled provision for the HVPs identified.

In this study, we identified a degree of variation in the extent to which demand for planned surgery within a community is met by the capacity of hospitals located in the same community. This is in addition to the current variation in waiting-list lengths and COVID-19 infection and hospitalisation rates. If variability could be reduced, or eliminated, then capacity planning is simplified. ${ }^{38}$ This strategy fits with NHS England's broader integration strategy as outlined in the Five Year Forward view and continued in the expansion of STPs to become larger integrated delivery systems.

The extent to which demand for surgical care will change as a result of COVID-19 remains uncertain. General practitioners and patients may prefer strategies of watchful waiting for minor surgical conditions, consequently reducing demand. Similarly, periods of lower community COVID-19 transmission may result in increased referral for surgical services before another wave of the pandemic takes hold. Regional variation in standardised rates of planned surgical procedures indicates that reductions in surgical demand may perhaps be greater in areas with lower pre-COVID-19 treatment thresholds and associated relative overuse. ${ }^{39}$

Similarly, the ability of hospitals to maintain preCOVID-19 surgical capacity during and after the pandemic is uncertain. Recent research has demonstrated that some endoscopy departments in England maintained or even increased activity during the COVID-19 pandemic, while others stopped services entirely. ${ }^{40}$ These findings indicate variation in local responses to the first wave of COVID-19 and allude to regional collaboration between surgical centres. In times of lower COVID-19 incidence, it could be expected that surgical supply may increase above preCOVID-19 rates through provision of additional operating theatre capacity in evenings and weekends or the involvement of private sector care providers. ${ }^{41}$ However, safely returning to baseline surgical capacity after a period of unprecedented disruption is a significant challenge in itself, and one where significant uncertainty remains. As a result of these uncertainties in future demand for, and supply of, surgical care, this study assumed future demand for planned surgical care would match historic demand from April 2017 to March 2018.

There are a number of limitations to the study. The COVID-19 epidemic is without precedent in recent history, so it was not possible to make substantially data-driven assumptions. The government has previously advised reducing national travel as a public health tool to limit COVID-19 spread. ${ }^{42}$ While our model does encourage patient mobility and could be criticised for the risk of further spread, it also facilitates more effective regional strategies to dedicate sites as COVID-19 clean or dirty. Stringent infection control measures will be an essential part of any reintroduction of routine services. Currently, there is mounting evidence that patients are not seeking out routine care due to the perceived risk of COVID-19 infection. ${ }^{43}$ There is therefore a possibility that patients 
will choose not to undergo any elective procedures in the current climate, nor travel to an unknown hospital for that care. Pooled waiting-lists are often disliked by surgeons who site the lack of autonomy and patient ownership with an increased risk of misdiagnosis, unnecessary procedures listed and unaddressed patient complexities. ${ }^{44} 45$ These risks can, and should, be mitigated by ensuring clear standardised patient pathways, patient triage and suitability assessments, clarity in the named responsible surgeon and pathways for ongoing continuity of care. Virtual platforms have become increasingly available during COVID-19 allowing remote consultation and triaging of patients prior to any procedures. ${ }^{46}$

This study included procedures of varying complexity and ability to increase surge capacity to overcome increased elective waiting-lists. Many of the most common procedures featured, including GI endoscopy, excisions of skin lesions and joint injection or aspiration, may be performed as 'day case' procedures and the ability to increase procedural throughput is less encumbered by the need for close anaesthetic support or high-dependency recovery space. In comparison, many higher risk procedures, including complex cardiac, cancer and orthopaedic surgery, are of lower volume. For example, during the study period, in England and Wales 16000 planned colorectal cancer resections were performed, while 14500 planned coronary artery bypass graft procedures were performed across the UK. ${ }^{47} 48$ These procedures are more likely to require significant anaesthetic support, postoperative critical or high-dependency care and lead to longer inpatient stays. Planning to retain capacity for these complex procedures may therefore entail a different approach to the pooled list approach suggested for the HVPs identified in this study.

Additionally, in using historical surgical volume as a means of quantifying maximum capacity, the study does not incorporate measures to increase surge capacity above prior maximal volumes. As such, the maximal capacities identified for pooled list communities in this study may significantly underestimate the throughput which may be achieved with additional measures to support expansion of surgical capacity.

Finally, while we have identified a mismatch between current policy (STP boundaries) and practice (the natural networks of surgical providers), we appreciate that implementation of new integrated networks on a larger scale would require significant new resources and planning. A new system of funding flows, mechanisms for regional waiting-list coordination and a cost per case mechanism or other financial incentive would be required to support this new model.

The NHS, despite being centrally funded, functions as a disparate collection of separate providers with their own priorities and resource constraints. In the COVID-19 pandemic, pre-existing structures of service delivery within the NHS were temporarily transformed. Primary care providers collaborated at a regional level to provide COVID-19 care through a network of hubs while hospitals collaborated with one another to ensure some cancer care could continue at a smaller number of 'clean' hospital sites. As health systems across the world look to address an ever-growing backlog for planned care created by COVID19 , this trend of enhanced collaboration must continue. If the NHS is to overcome this backlog and cope with further waves of COVID-19, providers of surgical care must develop the means by which they may share a collective case load for low-risk patients. What is certain is that the NHS, along with most other healthcare delivery systems, is having to make seismic changes to the way it works in order to best manage ongoing complexities. This study provides a solution with greater regional capacity flexibility with which to respond and adapt. Redesigning arbitrary geographical boundaries to follow expanded natural surgical community networks has the potential to increase efficiency by flexing existing supply to meet demand. This, in addition to other key strategies, could have a profound effect on tackling the massive backlog of cases accruing during this deadly pandemic, thereby preventing further death, disability and reduced productivity from delayed surgery.

Acknowledgements Data management was provided by the Big Data and Analytical Unit (BDAU) at the Institute of Global Health Innovation (IGHI), Imperial College London.

Collaborators The PanSurg Collaborative: Amish Acharya, Ravi Aggarwal, Jonathan Clarke, Max Denning, James Kinross, Sheraz Markar, Guy Martin, Sam Mason, Sanjay Purkayastha, Alasdair Scott, Viknesh Sounderajah, Jasmine Winter Beatty and Seema Yalamanchili

Contributors $\mathrm{JC}$ and $\mathrm{AM}$ were involved in all aspects of the study. MB was involved in the development of the methodology and assisted in the formal analysis. SRM, MB and JK were involved in the planning, conduct and reporting of the study. $\mathrm{JC}$ has had access to all the data in the study and all authors had final responsibility for the decision to submit for publication.

Funding This article is an independent research supported by grants from the Peter Sowerby Foundation and the National Institute for Health Research (NIHR) Imperial Patient Safety and Translational Research Centre (PSTRC_2016_004). Infrastructure support for this work was provided by the NIHR Imperial Biomedical Research Centre (BRC 1215-20013). MB and JC acknowledge the support from EPSRC grant EP/N014529/1 supporting the EPSRC Centre for Mathematics of Precision Healthcare. JC acknowledges support from the Wellcome Trust (215938/Z/19/Z). JK reports funding from H2020-ITN grant, NIHR-i4i and CRUK. Johnson \& Johnson has supported the activities of the PanSurg Collaborative with an educational grant.

Disclaimer The views expressed in this publication are those of the author(s) and not necessarily those of the NHS, the National Institute for Health Research or the Department of Health.

Map disclaimer The depiction of boundaries on this map does not imply the expression of any opinion whatsoever on the part of BMJ (or any member of its group) concerning the legal status of any country, territory, jurisdiction or area or of its authorities. This map is provided without any warranty of any kind, either express or implied.

Competing interests JK reports consultancy for Verb robotics/Ethicon and is a shareholder of OneWelbeck Day Surgery.

Patient consent for publication Not required.

Ethics approval This study received local ethical approval through the Imperial College Research Ethics Committee (17IC4178).

Provenance and peer review Not commissioned; externally peer reviewed.

Data availability statement Data used in this study were obtained from NHS Digital for the purpose of this work and may only be accessed through direct application to NHS Digital. Patient-level data are required for the analyses conducted, and therefore sharing of data pertaining to this study is not possible. Data for surgical community assignments are available from the authors on request. 
Supplemental material This content has been supplied by the author(s). It has not been vetted by BMJ Publishing Group Limited (BMJ) and may not have been peer-reviewed. Any opinions or recommendations discussed are solely those of the author(s) and are not endorsed by BMJ. BMJ disclaims all liability and responsibility arising from any reliance placed on the content. Where the content includes any translated material, BMJ does not warrant the accuracy and reliability of the translations (including but not limited to local regulations, clinical guidelines, terminology, drug names and drug dosages), and is not responsible for any error and/or omissions arising from translation and adaptation or otherwise.

Open access This is an open access article distributed in accordance with the Creative Commons Attribution 4.0 Unported (CC BY 4.0) license, which permits others to copy, redistribute, remix, transform and build upon this work for any purpose, provided the original work is properly cited, a link to the licence is given, and indication of whether changes were made. See: https://creativecommons.org/ licenses/by/4.0/.

\section{ORCID iDs}

Jonathan Clarke http://orcid.org/0000-0003-1495-7746

Sheraz Rehan Markar http://orcid.org/0000-0001-8650-2017

Mauricio Barahona http://orcid.org/0000-0002-1089-5675

\section{REFERENCES}

1 Turaga KK, Girotra S. Are we harming cancer patients by delaying their cancer surgery during the COVID-19 pandemic? Ann Surg 2020

2 Brindle ME, Doherty G, Lillemoe K, et al. Approaching surgical triage during the COVID-19 pandemic. Ann Surg 2020;272:e40-2.

3 American College of Surgeons. COVID-19: elective case triage guidelines for surgical care. Available: https://www.facs.org/covid19/clinical-guidance/elective-case

4 Royal College of Surgeons. COVID-19: good practice for surgeons and surgical teams. England: Royal College of Surgeons, 2020.

5 COVIDSurg Collaborative, Nepogodiev D, Bhangu A. Elective surgery cancellations due to the COVID-19 pandemic: global predictive modelling to inform surgical recovery plans. Br J Surg 2020

6 Brennan S. 'Mountain' waiting list will need five-year plan, says royal college. Health Services J 2020.

7 Brennan S. Trusts told to create 'covid-free hubs' for cancer patients. Health Services J 2020.

8 Kasivisvanathan V, Lindsay J, Rakhshani-moghadam S, et al. Evaluation of 30-day mortality for 500 patients undergoing nonemergency surgery in a COVID-19 cold site within a multicentre regional surgical network during the COVID-19 pandemic. medRxiv 2020.

9 National Audit Office. NHS waiting times for elective and cancer treatment. England: Department of Health and Social Care, 2019.

10 NHS. NHS waiting times for elective and cancer treatment. England: Department of Health \& Social Care, 2019.

11 Challen R T-AK, Pitt M, et al. Estimates of regional infectivity of COVID-19 in the United Kingdom following imposition of social distancing measures. medRxiv 2020.

12 Dixon ARR, Appleby J, Burge P. How patients choose and how providers respond. The King's Fund, 2010.

13 Dawson D, Gravelle H, Jacobs R, et al. The effects of expanding patient choice of provider on waiting times: evidence from a policy experiment. Health Econ 2007;16:113-28.

14 Service TaCD. National clinical coding standards OPCS-4. Accurate data for quality information health and social care information centre, 2019.

15 Quan H, Li B, Couris CM, et al. Updating and validating the Charlson comorbidity index and score for risk adjustment in hospital discharge abstracts using data from 6 countries. Am J Epidemiol 2011;173:676-82.

16 NHS Digital. Estates returns information collection, 2020.

17 Sinnott RW. Virtues of the harversine. Sky Telescope 1984;68:158.

18 Office for National Statistics. Lower layer super output areas population weighted Centroids, 2011. Available: https://geoportal. statistics.gov.uk/datasets/lower-layer-super-output-areas-december2011-population-weighted-centroids

19 Clarke JM, Barahona M, Darzi AW. Defining hospital catchment areas using multiscale community detection: a case study for planned orthopaedic care in England. bioRxiv 2019;619692.
20 Beguerisse-Díaz M, Vangelov B, Barahona M. Finding role communities in directed networks using Role-Based similarity, Markov stability and the relaxed minimum spanning tree. Paper presented at: 2013 IEEE global conference on signal and information processing; 3-5 Dec 2013, 2013.

21 Altuncu MT, Mayer E, Yaliraki SN, et al. From free text to clusters of content in health records: an unsupervised graph partitioning approach. Appl Netw Sci 2019;4:2.

22 Schaub MT, Delvenne J-C, Yaliraki SN, et al. Markov dynamics as a zooming lens for multiscale community detection: non clique-like communities and the field-of-view limit. PLoS One 2012;7:e32210.

23 Lambiotte R, Delvenne J-C, Barahona M. Random walks, Markov processes and the multiscale modular organization of complex networks. IEEE Trans Netw Sci Eng 2014;1:76-90.

24 Rhoades SA. Market share inequality, the $\mathrm{HHI}$, and other measures of the firm-composition of a market. Rev Ind Organ 1995;10:657-74.

25 Martin S, Rice N, Jacobs R, et al. The market for elective surgery: joint estimation of supply and demand. $J$ Health Econ 2007;26:263-85.

26 NHS England. Advice on maintaining cancer treatment during the COVID-19 response. Improvement NEaN, 2020.

27 PanSurg. Exclusive: the three vital lessons Italian hospitals have learned in fighting covid-19. Health Services J 2020.

28 Ballini L, Negro A, Maltoni S, et al. Interventions to reduce waiting times for elective procedures. Cochrane Database Syst Rev 2015;2:CD005610.

29 Leach P, Rutherford SA, King AT, et al. Generic waiting lists for routine spinal surgery. J $R$ Soc Med 2004;97:119-20.

30 John Appleby AH, Devlin N. What is the real cost of more patient choice? The King's Fund, 2003.

31 Madsen M, Asmundsson P, Brekke IB, et al. Scandiatransplant: thirty years of cooperation in organ transplantation in the Nordic countries. Clin Transpl 1998:121-31.

32 Siciliani L, Martin S. An empirical analysis of the impact of choice on waiting times. Health Econ 2007;16:763-79.

33 Dawson D, Jacobs R, Martin S, et al. Is patient choice an effective mechanism to reduce waiting times? Appl Health Econ Health Policy 2004;3:195-203.

34 Dixon A. New labour's market reforms - patient choice of hospital, 2011.

35 Damiani M, Propper C, Dixon J. Mapping choice in the NHS: cross sectional study of routinely collected data. BMJ 2005;330:284.

36 Ryan M, Mclntosh E, Dean T, et al. Trade-offs between location and waiting times in the provision of health care: the case of elective surgery on the Isle of Wight. J Public Health Med 2000;22:202-10.

37 Aggarwal A, Lewis D, Mason M, et al. Effect of patient choice and hospital competition on service configuration and technology adoption within cancer surgery: a national, population-based study. Lancet Oncol 2017;18:1445-53.

38 Pandit JJ, Pandit M, Reynard JM. Understanding waiting lists as the matching of surgical capacity to demand: are we wasting enough surgical time? Anaesthesia 2010;65:625-40.

39 John Appleby VR, Frosini F, Bevan G, et al. Variations in health care. The King's Fund. Available: https://www.kingsfund.org.uk/sites/ default/files/field/field_publication_file/Variations-in-health-caregood-bad-inexplicable-report-The-Kings-Fund-April-2011.pdf

40 Markar SR, Clarke J, Kinross J, et al. Practice patterns of diagnostic upper gastrointestinal endoscopy during the initial COVID-19 outbreak in England. Lancet Gastroenterol Hepatol 2020;5:804-5.

41 Permain N. COVID-19: update on partnership working with the independent sector providers and the independent healthcare providers network (IHPN) in: teams RDRL, 2020.

42 Government UK. The health protection (coronavirus, restrictions) (England) regulations 2020, 2020

43 Owens B. Reduced attendances and admissions: lessons from the covid crisis. Health Services J 2020.

44 Ramchandani M, Mirza S, Sharma A, et al. Pooled cataract waiting lists: views of hospital consultants, general practitioners and patients. J R Soc Med 2002;95:598-600.

45 British Orthopaedic Association. Position statement on pooled waiting lists [press release], 2013.

46 NHS England. Elective care transformation programme, 2020.

47 National Institute for Cardiovascular Outcomes Research. National adult cardiac surgery audit, 2019 summary report (2015/16-2017/18 data), 2019.

48 National Bowel Cancer Audit. National bowel cancer audit, Annual report 2019. An audit of the care received by people with bowel cancer in England and Wales, 2019. 